\title{
Generalization of geometric phase to completely positive maps
}

\author{
Marie Ericsson and Erik Sjöqvist \\ Department of Quantum Chemistry, Uppsala University, Box 518, Se-751 20, Sweden \\ Johan Brännlund \\ Stockholm University, SCFAB, Fysikum, Se-106 91 Stockholm, Sweden \\ Daniel K.L. Oi \\ Centre for Quantum Computation, Clarendon Laboratory, \\ University of Oxford, Parks Road, Oxford OX1 3PU, UK \\ Arun K. Pati \\ Institute of Physics, Bhubaneswar-751005 Orissa, India
}

\begin{abstract}
We generalize the notion of relative phase to completely positive maps with known unitary representation, based on interferometry. Parallel transport conditions that define the geometric phase for such maps are introduced. The interference effect is embodied in a set of interference patterns defined by flipping the environment state in one of the two paths. We show for the qubit that this structure gives rise to interesting additional information about the geometry of the evolution defined by the CP map.
\end{abstract}

Berry's [1] discovery of a geometric phase accompanying cyclic adiabatic evolution has triggered an immense interest in holonomy effects in quantum mechanics and has led to many generalizations. The restriction of adiabaticity was removed by Aharonov and Anandan [2], who pointed out that the geometric phase is due to the curvature of the projective Hilbert space. It was extended to noncyclic evolution by Samuel and Bhandari [3] (see also Refs. [4]), based on Pancharatnam's [5] work on interference of classical light in distinct states of polarization. Another development of geometric phase was initiated by Uhlmann [6] who introduced this notion to mixed quantal states. More recently another mixed state geometric phase in the particular case of unitary evolution was discovered in the context of interferometry [7].

The geometric phase has shown to be useful in the context of quantum computing as a tool to achieve faulttolerance [8]. For practical implementations of geometric quantum computing, it is important to understand the behavior of the geometric phase in the presence of decoherence. For this, we generalize in this Letter the idea in [7] to completely positive (CP) maps, i.e. we define the relative (Pancharatnam) phase and introduce a notion of parallel transport with concomitant geometric phase for such maps. These generalized concepts reduces to that of [7] in the case of unitary evolutions.

Let us first consider a Mach-Zehnder interferometer with a variable relative $U(1)$ phase $\chi$ in one of the interferometer beams (the reference beam) and assume that the interfering particles carry an additional internal degree of freedom, such as spin or polarization, in a pure state $|k\rangle$. The other beam (the target beam) is exposed to the unitary operator $U_{i}$, yielding the output interference pattern $I \propto 1+\nu \cos (\chi-\alpha)$, which is completely determined by the complex quantity

$$
\nu e^{i \alpha}=\left\langle k\left|U_{i}\right| k\right\rangle .
$$

Thus, by varying $\chi$, the relative phase $\alpha$ and visibility $\nu$ can be distinguished experimentally. We note that $\alpha$ is a shift in the maximum of the interference pattern, a fact that motivated Pancharatnam [5] to define it as the relative phase between the internal states $|k\rangle$ and $U_{i}|k\rangle$ of the two beams.

Pancharatnam's analysis was generalized in [7] to mixed states undergoing unitary evolution as follows. Assume that the incoming particle is in a mixed internal state $\rho=\sum_{k=1}^{N} w_{k}|k\rangle\langle k|$, where $N$ is the dimension of the internal Hilbert space. Each pure component $|k\rangle$ of this mixture contributes an interference profile given by $\left\langle k\left|U_{i}\right| k\right\rangle=\nu_{k} e^{i \alpha_{k}}$ weighted by the its probability $w_{k}$ yielding $I=\sum_{k} w_{k} I_{k} \propto 1+\sum_{k} w_{k} \nu_{k} \cos \left[\chi-\alpha_{k}\right]$. Noting that $\operatorname{Tr}\left(U_{i} \rho\right)=\sum w_{k}\left\langle k\left|U_{i}\right| k\right\rangle$, this can also be written as $I \propto 1+\left|\operatorname{Tr}\left(U_{i} \rho\right)\right| \cos \left[\chi-\arg \operatorname{Tr}\left(U_{i} \rho\right)\right]$. The key result is that the interference fringes, produced by varying the phase $\chi$, is shifted by $\alpha=\arg \operatorname{Tr}\left(U_{i} \rho\right)$ and that this shift reduces to Pancharatnam's original prescription for pure states. These two facts are the central properties for $\alpha$ being a natural generalization of Pancharatnam's relative phase to mixed states undergoing unitary evolution. Furthermore, it is clear that the quantity that extends that of Eq. (11) to mixed states is

$$
\nu e^{i \alpha}=\operatorname{Tr}\left(U_{i} \rho\right)
$$

with visibility $\nu=\left|\operatorname{Tr}\left(U_{i} \rho\right)\right|$.

Nonunitary evolution of a quantal state may be conveniently modeled by appending an environment in a pure state that we designate $\left|0_{e}\right\rangle$, i.e.

$$
\varrho=\rho \otimes\left|0_{e}\right\rangle\left\langle 0_{e}\right|,
$$




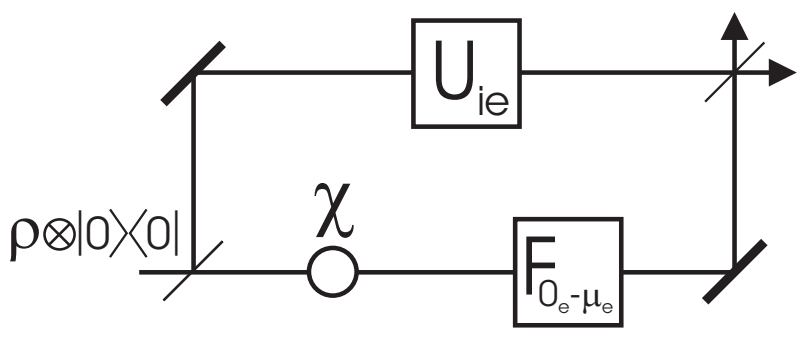

FIG. 1: Interferometer for determining complete interference information.

and letting the combined state evolve unitarily as $\varrho \rightarrow$ $\varrho^{\prime}=U_{i e} \varrho U_{i e}^{\dagger}$ with given $U_{i e}$. The evolved density matrix of the internal part is obtained by tracing over this environment yielding

$$
\rho^{\prime}=\operatorname{Tr}_{e} \varrho^{\prime}=\sum_{\mu} m_{\mu} \rho m_{\mu}^{\dagger},
$$

where the Kraus operators are $m_{\mu}=\left\langle\mu_{e}\left|U_{i e}\right| 0_{e}\right\rangle$ [9] in terms of an orthonormal basis $\left\{\left|\mu_{e}\right\rangle\right\}, \mu=0, \ldots, K-1 \geq$ $N$, of the $K$-dimensional Hilbert space of the environment. This map $\Lambda$ is completely positive $(\mathrm{CP})$, i.e. it takes density operators into density operators, and all trivial extensions $I \otimes \Lambda$ likewise. Conversely, any CP

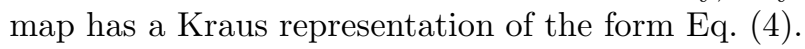

Using Eq. (2), the interference pattern for the incoming state in Eq. (3), evolved with $U_{i e}$ in the target beam, is described by

$$
\nu_{0} e^{i \alpha_{0}}=\operatorname{Tr}_{i e}\left[U_{i e} \varrho\right]=\operatorname{Tr}_{i}\left[m_{0} \rho\right],
$$

where we have used $\left\langle 0_{e} \mid \mu_{e}\right\rangle=\delta_{0 \mu}$. The quantity $\alpha_{0}$ is a natural definition of relative phase as it shifts the maximum of the interference pattern and reduces to the phase defined in [7] for unitarily evolving mixed states.

Since phase information has leaked from the system part, the interference information contained in Eq. (5) is only partial. The remaining part may be uncovered by flipping the state of the environment associated with the reference beam to an orthogonal state $\left|\mu_{e} \neq 0_{e}\right\rangle$ (Fig. 1) [12]. This transformation may be represented by the operator

$$
U=\left(\begin{array}{ll}
0 & 0 \\
0 & 1
\end{array}\right) \otimes U_{i e}+\left(\begin{array}{rr}
e^{i \chi} & 0 \\
0 & 0
\end{array}\right) \otimes 1_{i} \otimes F_{0_{e} \rightarrow \mu_{e}}
$$

where the first matrix in each term represents the spatial part and the operator $F_{0_{e} \rightarrow \mu_{e}}$ flips $\left|0_{e}\right\rangle$ to $\left|\mu_{e} \neq 0_{e}\right\rangle$. Fig. 2 shows the equivalent quantum network. The interference pattern is determined by

$$
\begin{aligned}
\nu_{\mu} e^{i \alpha_{\mu}} & =\operatorname{Tr}_{i e}\left[U_{i e} \rho\left|0_{e}\right\rangle\left\langle 0_{e}\right| F_{0_{e} \rightarrow \mu_{e}}^{\dagger}\right] \\
& =\operatorname{Tr}_{i e}\left[U_{i e} \rho\left|0_{e}\right\rangle\left\langle\mu_{e}\right|\right]=\operatorname{Tr}_{i}\left[m_{\mu} \rho\right]
\end{aligned}
$$

for each $\mu=1, \ldots, K-1$. The set $\left\{\nu_{\mu} e^{i \alpha_{\mu}}\right\}, \mu=$ $0, \ldots, K-1$, contains maximal information about the

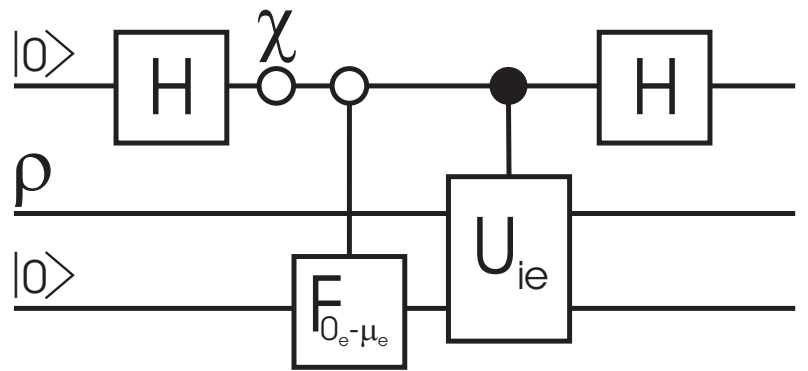

FIG. 2: Quantum network for interferometry of a state undergoing a CP map.

interference effect associated with the CP map, by measuring on the system alone. For unitarily evolving mixed states one obtains $\nu_{\mu}=\delta_{0 \mu}$, due to orthogonality of the environmental states, and the surviving interference pattern Eq. (5) reduces to that of [7].

The results above can be derived by considering purifications. We may lift $\varrho$ in Eq. (3) to a purified state $|\Psi\rangle$ by attaching an ancilla according to

$$
|\Psi\rangle=\sum_{k} \sqrt{w_{k}}\left|k_{i}\right\rangle\left|0_{e}\right\rangle\left|k_{a}\right\rangle
$$

with $\left\{\left|k_{a}\right\rangle\right\}$ a basis in an auxiliary Hilbert space of dimension at least as large as that of the internal Hilbert space. This state is mapped by the operators $U=U_{i e} \otimes I_{a}$ and $F=I_{i} \otimes F_{0_{e} \rightarrow \mu_{e}} \otimes I_{a}$ in the target and reference beam, respectively, i.e.

$$
\begin{aligned}
\left|\Psi_{\mathrm{tar}}\right\rangle & =\sum_{k} \sqrt{w_{k}}\left[U_{i e}\left|k_{i}\right\rangle\left|0_{e}\right\rangle\right]\left|k_{a}\right\rangle, \\
\left|\Psi_{\mathrm{ref}}\right\rangle & =\sum_{k} \sqrt{w_{k}}\left|k_{i}\right\rangle\left[F_{0_{e} \rightarrow \mu_{e}}\left|0_{e}\right\rangle\right]\left|k_{a}\right\rangle,
\end{aligned}
$$

Their inner-product becomes

$$
\begin{aligned}
\left\langle\Psi_{\mathrm{ref}} \mid \Psi_{\mathrm{tar}}\right\rangle= & \sum_{k} w_{k}\left\langle k_{i}\left|\left\langle 0_{e}\left|F_{0_{e} \rightarrow \mu_{e}}^{\dagger} U_{i e}\right| 0_{e}\right\rangle\right| k_{i}\right\rangle \\
& =\sum_{k} w_{k}\left\langle k_{i}\left|m_{\mu}\right| k_{i}\right\rangle=\operatorname{Tr}_{i}\left(m_{\mu} \rho\right)
\end{aligned}
$$

in agreement with Eqs. (5) and (7).

To illustrate the above, let us consider the depolarization channel [10] acting on a qubit in the initial state $\rho=\frac{1}{2}(I+\boldsymbol{r} \cdot \boldsymbol{\sigma})$, where $\boldsymbol{r}=(x, y, z)$ is the Bloch vector with the length $|\boldsymbol{r}| \leq 1, \boldsymbol{\sigma}=\left(\sigma_{x}, \sigma_{y}, \sigma_{z}\right)$ are the standard Pauli matrices, and $I$ is the $2 \times 2$ unit matrix. We will model this with the Kraus operators

$$
\begin{array}{ll}
m_{0}=\sqrt{1-p} I, & m_{1}=\sqrt{p / 3} \sigma_{x}, \\
m_{2}=\sqrt{p / 3} \sigma_{y}, & m_{3}=\sqrt{p / 3} \sigma_{z}
\end{array}
$$

that map $\rho \rightarrow \rho^{\prime}=\frac{1}{2}\left(I+\boldsymbol{r}^{\prime} \cdot \boldsymbol{\sigma}\right)$. Here, $m_{1}, m_{2}$, and $m_{3}$, correspond to bit flip, both bit and phase flip, and phase flip, respectively. $p$ is the probability that one 
of these errors occurs and it determines the shrinking factor $\left|\boldsymbol{r}^{\prime}\right| /|\boldsymbol{r}|=(1-4 p / 3)$ of the Bloch vector. Exposing the depolarization channel to one of the interferometer beams, the interference pattern is determined by Eq. (5) as

$$
\nu_{0} e^{i \alpha_{0}}=\operatorname{Tr}_{i}\left(\rho m_{0}\right)=\sqrt{1-p}
$$

This quantity is real and positive so that $\alpha_{0}=0$, thus the channel only reduces the visibility by the factor $\sqrt{1-p}$. If the state of the environment is flipped to any of the other $\left|\mu_{e}\right\rangle$ 's the interference pattern Eq. (7) is determined by

$$
\nu_{\mu} e^{i \alpha_{\mu}}=\operatorname{Tr}_{i}\left(\rho m_{\mu}\right)=\sqrt{\frac{p}{3}} r_{\mu}
$$

where $r_{\mu}=x, y, z$ for $\mu=1,2,3$, respectively. These are also real and positive so that $\alpha_{\mu}=0$. The only effect is a nonvanishing visibility proportional to the probability amplitude $\sqrt{p / 3}$ associated with the corresponding error. The absence of phase shifts can be understood from the fact that the depolarization channel only shrinks the length of the Bloch vector.

Another illustration of the general formalism above is provided by the amplitude damping channel [10], which models, e.g., the decay of an atom from one of its excited states to its ground state by emitting a photon. It may be described by the Kraus operators

$$
\begin{aligned}
& m_{0}=\frac{1}{2}\left(I+\sigma_{z}\right)+\frac{\sqrt{1-p}}{2}\left(I-\sigma_{z}\right), \\
& m_{1}=\frac{\sqrt{p}}{2}\left(\sigma_{x}+i \sigma_{y}\right),
\end{aligned}
$$

where $p$ is the decay probability. When no photon has been emitted in the reference beam the interference pattern is determined by

$$
\nu_{0} e^{i \alpha_{0}}=\operatorname{Tr}_{i}\left(\rho m_{0}\right)=\frac{1}{2}(1+\sqrt{1-p}+z[1-\sqrt{1-p}]),
$$

which is real and positive so that $\alpha_{0}=0$, and only the visibility $\nu_{0}$ is affected anisotropically by the channel. On the other hand, if a photon has been emitted in the reference beam, we obtain

$$
\nu_{1} e^{i \alpha_{1}}=\operatorname{Tr}_{\mathrm{i}}\left(\rho m_{1}\right)=\frac{\sqrt{p}}{2}(x+i y)
$$

being dependent only upon the $x-y$ projection of the initial Bloch vector. Here, the visibility decrease is proportional to the decay probability amplitude $\sqrt{p}$ and there is a shift in the interference oscillations determined by the angle of the $x-y$ projection to the $x$ axis. By checking whether or not there is a photon in the reference beam it should be possible experimentally to distinguish the two interference patterns determined by Eqs. (15) and (16) by post-selection.
In the general case, it is convenient to make a polar decomposition of $m_{\mu}$ such that

$$
m_{\mu}=h_{\mu} u_{\mu}
$$

where $h_{\mu}$ is Hermitian and positive, and $u_{\mu}$ is unitary. The action of each $m_{\mu}$ is uniquely defined up to $N$ phase factors by the evolution of the system's density operator. This ambiguity must be associated with the corresponding unitarity $u_{\mu}$ as the Hermitian part is unique.

Let us clarify this point for a qubit, where we may write $u_{\mu}=e^{-i \theta_{\mu} \boldsymbol{e}_{\mu} \cdot \boldsymbol{\sigma}}$ and $h_{\mu}=a_{\mu}+\boldsymbol{b}_{\mu} \cdot \boldsymbol{\sigma}$. It is possible to evaluate Eq. (7) for $\rho=\frac{1}{2}(I+\boldsymbol{r} \cdot \boldsymbol{\sigma})$ yielding

$$
\begin{aligned}
\nu_{\mu} e^{i \alpha_{\mu}}= & \left(a_{\mu}+\boldsymbol{r} \cdot \boldsymbol{b}_{\mu}\right) \cos \theta_{\mu}+\left(\boldsymbol{r} \times \boldsymbol{e}_{\mu}\right) \cdot \boldsymbol{b}_{\mu} \sin \theta_{\mu} \\
& -i\left(\boldsymbol{e}_{\mu} \cdot \boldsymbol{b}_{\mu}+a_{\mu} \boldsymbol{r} \cdot \boldsymbol{e}_{\mu}\right) \sin \theta_{\mu} .
\end{aligned}
$$

Now, with $\boldsymbol{r}=\boldsymbol{r} \boldsymbol{n}$ the density operator is unaffected by the change $u_{\mu} \rightarrow u_{\mu} e^{-i \gamma_{\mu} \boldsymbol{n} \cdot \boldsymbol{\sigma}}$ as the additional part commutes with $\rho$. However, the interference pattern is determined by this new unitarity and therefore changes according to

$$
\begin{aligned}
\cos \theta_{\mu} \rightarrow & \cos \tilde{\theta}_{\mu}=\cos \theta_{\mu} \cos \gamma_{\mu}-\boldsymbol{e}_{\mu} \cdot \boldsymbol{n} \sin \theta_{\mu} \sin \gamma_{\mu} \\
\boldsymbol{e}_{\mu} \sin \theta_{\mu} \rightarrow & \tilde{\boldsymbol{e}}_{\mu} \sin \tilde{\theta}_{\mu}=\boldsymbol{n} \cos \theta_{\mu} \sin \gamma_{\mu} \\
& +\boldsymbol{e}_{\mu} \sin \theta_{\mu} \cos \gamma_{\mu}+\boldsymbol{e}_{\mu} \times \boldsymbol{n} \sin \theta_{\mu} \sin \gamma_{\mu}(19)
\end{aligned}
$$

where $u_{\mu} e^{-i \gamma_{\mu} \boldsymbol{n} \cdot \boldsymbol{\sigma}}=e^{-i \tilde{\theta}_{\mu} \tilde{\boldsymbol{e}}_{\mu} \cdot \boldsymbol{\sigma}}$.

The phase ambiguity can be removed by introducing a notion of parallel transport and concomitant geometric phases for a continuous (time) parametrization of the CP map. For each interference pattern, the $N$ parallel transport conditions read

$$
\left\langle k\left|\tilde{u}_{\mu}^{\dagger}(t) \dot{\tilde{u}}_{\mu}(t)\right| k\right\rangle=0, \quad k=1, \ldots N
$$

where we have decomposed $m_{\mu}(t)=h_{\mu}(t) u_{\mu}(t)=$ $h_{\mu}(t) v_{\mu} \tilde{u}_{\mu}(t)$ with $v_{\mu}=u_{\mu}(0)$ completely specified by the channel, $\tilde{u}_{\mu}(0)=1$, and we have assumed $h_{\mu}(0)=\delta_{\mu 0}$. These conditions naturally extend those in Eq. (13) of [7] to the case of CP maps. They are sufficient and necessary to arrive at a unique notion of geometric phase in the context of single-particle interferometry. The set of these geometric phases for $\mu=0, \ldots, K-1$ provides the complete geometric picture of the CP map in interferometry, given the unitary representation.

Now, let us assume for simplicity that $h_{\mu}$ and $\rho$ diagonalize in the same basis $\{|k\rangle\}$ for all $t \geq 0$. In the cyclic case, where for each $k$ we have $\tilde{u}_{\mu}:|k\rangle \rightarrow e^{i \beta_{k}^{\mu}}|k\rangle, \beta_{k}^{\mu}$ being the corresponding cyclic pure state geometric phase, the interference pattern is given by

$$
\nu_{\mu} e^{i \Phi_{\mu}}=\sum_{k} w_{k}\left\langle k\left|h_{\mu}\right| k\right\rangle\left\langle k\left|v_{\mu}\right| k\right\rangle e^{i \beta_{k}^{(\mu)}},
$$

where all $\left\langle k\left|h_{\mu}\right| k\right\rangle \geq 0$ are real-valued as $h_{\mu}$ is Hermitian and positive. 
Let us use Eq. (21) to compute the geometric phase for a qubit with $r \neq 0$ in the depolarization channel with a unitary rotation added. Thus, we replace the Kraus operators $m_{\mu}$ in Eq. (11) by $m_{\mu} \tilde{u}$ with $\tilde{u}$ being an $S U(2)$ operator that fulfills the parallel transport conditions Eq. (20). Here, $h_{\mu}$ is diagonal for all Kraus operators, $\left(v_{1}, v_{2}, v_{3}\right)=\left(\sigma_{x}, \sigma_{y}, \sigma_{z}\right)$, and $\tilde{u}_{\mu}=\tilde{u}$ for all $\mu$. For cyclic $\tilde{u}$, the interference patterns are determined by

$$
\begin{aligned}
& \nu_{0} e^{i \Phi_{0}}=\sqrt{1-p}(\cos (\Omega / 2)+i r \sin (\Omega / 2)) \\
& \nu_{1} e^{i \Phi_{1}}=0 \\
& \nu_{2} e^{i \Phi_{2}}=0 \\
& \nu_{3} e^{i \Phi_{3}}=\sqrt{p / 3}(r \cos (\Omega / 2)+i \sin (\Omega / 2))
\end{aligned}
$$

with $\Omega$ the solid angle enclosed by the loop on the Bloch sphere. The first interference pattern is precisely that obtained in [] modified by a visibility factor $\sqrt{1-p} . \nu_{1}$ and $\nu_{2}$ vanish since the corresponding errors involve bit flips. Surprisingly, the pure phase flip in the last interference pattern introduces a nontrivial change in the appearance of $r$. This is due to the fact that this phase flip introduces an additional relative sign between the weights of the pure state interference patterns and is purely an effect of the decoherence.

We may also consider the case where $\tilde{u}$ takes $|0\rangle \rightarrow$ |1) and vice versa. Any such $\tilde{u}$ fulfilling the parallel transport conditions Eq. (20) may be written as $e^{-i(\pi / 2)\left[\cos \varphi \sigma_{x}+\sin \varphi \sigma_{y}\right]}$. Here, only the errors containing bit flips produce a nonvanishing interference effect that can be interpreted geometrically by noting that the $S U(2)$ error operators $i v_{1}$ and $i v_{2}$ are themselves taking $|0\rangle \rightarrow|1\rangle$ and $|1\rangle \rightarrow|0\rangle$ along geodesics intersecting the $y$ and $x$ axis, respectively. Thus, $i v_{1} \tilde{u}$ defines a closed loop on the Bloch sphere that encloses the solid angle $2 \pi-2 \varphi$, yielding

$$
\begin{aligned}
\nu_{1} e^{i \Phi_{1}} & =-i \sqrt{\frac{p}{3}}\left[\frac{1+r}{2}\left\langle 0\left|i v_{1} \tilde{u}\right| 0\right\rangle+\frac{1-r}{2}\left\langle 1\left|i v_{1} \tilde{u}\right| 1\right\rangle\right] \\
& =\sqrt{\frac{p}{3}} e^{-i \pi / 2}(\cos \varphi+i r \sin \varphi)
\end{aligned}
$$

Similarly, $i v_{2} \tilde{u}$ defines the solid angle $3 \pi-2 \varphi$, so that the interference pattern is determined by

$$
\begin{aligned}
\nu_{2} e^{i \Phi_{2}} & =-i \sqrt{\frac{p}{3}}\left[\frac{1+r}{2}\left\langle 0\left|i v_{2} \tilde{u}\right| 0\right\rangle+\frac{1-r}{2}\left\langle 1\left|i v_{2} \tilde{u}\right| 1\right\rangle\right] \\
& =\sqrt{\frac{p}{3}} e^{-i \pi}(r \cos \varphi+i \sin \varphi)
\end{aligned}
$$

Again, there is a nontrivial change in the appearance of $r$ in the last expression due to the fact that this error also contains a phase flip.

To summarize, we have provided a generalization of the notion of relative phase to completely positive maps with known unitary representation, based on interferometry. We have further introduced parallel transport conditions that define the geometric phase for such maps. The interference effect is embodied in a set of interference patterns defined by flipping the environment state in one of the two particle beams. We have shown in the qubit case that this structure gives rise to interesting additional information about the geometry of the evolution defined by the CP map. We hope that this work will trigger new experiments on geometric phases for quantal systems exposed to environmental interactions.

We would like to thank Artur Ekert for useful discussions. The work by E.S. was financed by the Swedish Research Council. D.K.L.O acknowledges the support of CESG (UK) and QAIP grant IST-1999-11234.

Note added: After completing this work, it has come to our knowledge that Peixoto de Faria et al. [11 have arrived at the interference pattern described by Eq. (5) in the context of measurement theory.

[1] M.V. Berry, Proc. R. Soc. London Ser. A 392, 45 (1984).

[2] Y. Aharonov and J.S. Anandan, Phys. Rev. Lett. 58, 1593 (1987).

[3] J. Samuel and R. Bhandari, Phys. Rev. Lett. 60, 2339 (1988).

[4] I.J.R. Aitchison and K Wanelik, Proc. R. Soc. London Ser. A 439, 25 (1992); N. Mukunda and R. Simon, Ann. Phys. (N.Y.) 228, 205 (1993).

[5] S. Pancharatnam, Proc. Indian Acad. Sci. A 44, 247 (1956).

[6] A. Uhlmann, Rep. Math. Phys. 24, 229 (1986).

[7] E. Sjöqvist, A.K. Pati, A. Ekert, J.S. Anandan, M. Ericsson, D.K.L. Oi, and V. Vedral, Phys. Rev. Lett. 85, 2845 (2000).

[8] J.A. Jones, V. Vedral, A. Ekert and G. Castagnoli, Nature 403, 869 (1999); A. Ekert, M. Ericsson, P. Hayden, H. Inamori, J.A. Jones, D.K.L. Oi, and V. Vedral, J. Mod. Opt. 47, 2501 (2000); G. Falci, R. Fazio, G.M. Palma, J. Siewert, V. Vedral, Nature 407, 355 (2000); W. Xiang-Bin, M. Keiji, Phys. Rev. Lett. 87, 097901 (2001); A. Nazir, T. P. Spiller, W. J. Munro, Phys. Rev. A 65, 042303 (2002).

[9] K. Kraus, States, Effects and Operations (SpringerVerlag, Berlin, 1983).

[10] J. Preskill, "Lecture notes for a course Information for Physics 219/ Computer Science 219, Quantum Computation," www.theory.caltech.edu/people/preskill/ph229.

[11] J.G. Peixoto de Faria, A.F.R. de Toledo Piza, and M.C. Nemes, e-print quant-ph/0205146.

[12] This assumes we have full control over the environment and that it is, e.g., an extra degree of internal freedom. 